\title{
HOMOGENEOUS MODELS IN GENERAL RELATIVITY AND GAS DYNAMICS
}

\author{
O. I. BOGOYAVLENSKII AND S. P. NOVIKOV
}

Dedicated to the founder of the Soviet school of topology

Pavel Sergeevich Aleksandrov

\begin{abstract}
The paper begins with a short survey of results on non-trivial models (that is, those that are not integrable analytically) in general relativity and gas dynamics. The investigation of these models is carried out by the methods of the qualitative theory of many-dimensional dynamical systems, using geometrical and topological ideas. The first section deals with the results of research on the evolution of homogeneous cosmological models with a hydrodynamic energy tensor-the impulse about a singularity. In the second section similar models are applied to the study of the complex oscillating regimes of a classical ideal compressible fluid. The Appendix contains new, unpublished results due to one of the authors, describing stochastic perturbation of a completely integrable Toda chain.
\end{abstract}

\section{INTRODUCTION}

Very few exact solutions are known either in general relativity or in gas dynamics. The best known of them are the homogeneous and isotropic solutions of Friedmann in relativistic cosmology, the spherically symmetric solutions of Schwarzschild and Tolman, and solutions by Kerr, Taub, Kazner etc. Approximately similar solutions are known also for three-dimensional problems of gas dynamics in the absence of viscosity. All the known exact solutions have a high degree of symmetry. However, they turn out to be unstable under compression of the material.

Anisotropic homogeneous models present a broader class of solutions, and the investigation of them allows us to see the development of these instabilities. Since homogeneous isotropic models are non-integrable both in general relativity and in gas dynamics, their investigation demands the use of contemporary methods of the qualitative theory of dynamical systems, making essential use of a number of geometrical and topological ideas. The regimes arising here have very complex characteristics, including stochastic ones.

A programme for the application of qualitative methods in problems of general relativity was developed in the authors' papers [1]-[4]; the application of these methods to the problem of the motion of a gravitating gas ellipsoid was accomplished in the papers [5] and [6] of one of the authors.

Over the past ten years fairly extensive literature has been devoted to homogeneous models in general relativity, not using the qualitative theory (see the bibliography in [7]). Firstly, a non-trivial regime with stochastic properties was discovered

Date: Received by the Editors 24 March 1976.

Translated by D. W. Jordan. 
by Belinskii, Lifshits, and Khalatnikov (the BLK oscillating regime, see [8]). Misner [9] was the first to apply the Hamiltonian formalism in these problems. Collins apparently first used the two-dimensional qualitative theory of Poincaré-Bendixson to study special (mainly axially symmetric) cosmological models $[10]^{1}$ some results on the early stages of evolution of the universe parallel with those of the present authors have been obtained by I. D. Novikov, Doroshkevich, and Lukash [11]. Despite the absence in these papers of an exact statement of the problem on typical states of the universe in the early stages of evolution, and despite the fact that their methods are approximate (they should rather be called analytical and numerical experiments), nonetheless a substantial number of the conclusions in [11] agree with results obtained at the same time by the present authors [2]. A detailed account of our results is given in $\S 1$ of this survey.

In the theory of an ideal compressible gas there are classes of solutions that are in many respects like the homogeneous models of general relativity. (This was pointed out to the authors by Anisimov and Zel'dovich.) In these solutions the velocities of the gas are linear functions of the coordinates. The description of the motion of a gas is formally equivalent to that of the motion of material point on the group of matrices $\mathrm{GL}(3, R)$ with a certain potential. Spherically symmetric solutions were found by Sedov, among them solutions of this type [15]. Some broader classes of solutions are investigated in $[16]-[19] .{ }^{2}$ Under compression, a spherically symmetric regime loses stability, and oscillations are set up. In the purely gas-dynamical problem any finite number of oscillations may occur, which are then replaced by an infinite expansion. When the particles have gravitational reciprocity, there arises a complex non-linear oscillating regime partially resembling a BLK regime. These results are given in $\S 2$.

The appendix "Oscillating regimes in systems with an exponential potential" gives new results due to one of the authors.

\section{$\S 1$. The Qualitative theory of homogeneous Cosmological models}

I. The general theory of relativity, as is well-known, studies four-dimensional space-time manifolds $M^{4}$ with the Einstein metric $g_{i j}(i, j=0,1,2,3)$, satisfying the Einstein equation

$$
R_{i j}-\frac{1}{2} g_{i j} R=\frac{8 \pi k}{c^{4}} T_{i j}
$$

Homogeneous cosmological models are defined by the fact that $M^{4}$ admits a group of motions $G$ (acting on the right, to be definite) with three-dimensional space-like orbits (that is, on an orbit the metric is negative). As a rule, the case taken is the simplest hydrodynamical energy-impulse tensor of matter

$$
T_{i j}=(p+\varepsilon) u_{i} u_{j}-p g_{i j}
$$

\footnotetext{
${ }^{1}$ In recent years the methods of the two-dimensional qualitative theory of Poincaré-Bendixson have also been applied in [12]-[14].

${ }^{2}$ We do not consider here the singular case of an incompressible liquid, to which a large classical literature [20], [21] is devoted; even the equations have a different form. For a compressible gas these equations were apparently first described and discussed by Ovsyannikov [16], the Hamiltonian form of these equations was indicated by Dyson [17].
} 
where $\varepsilon$ is the energy density, $p$ the pressure, $p=k \varepsilon, 0 \leqslant k<1, u^{i}$ is the 4 -velocity of the matter, and the frame of reference is said to be synchrone, that is, $g_{00}=1$, $g_{0 \alpha}=0$. We suppose that the system of units is such that $c=8 \pi k / c^{4}=1$.

The open models of Bianchi type VII and VIII and the closed model of type IX, where $G=\mathrm{SU}(2)$, are the most complex ones. Let us look in more detail at this case, assuming in addition that the material does not move in the medium; that is, $u_{i}=\delta_{i 0}$.

Under these assumptions there exists a basis of right-invariant vector fields $X_{1}, X_{2}, X_{3}$ on the orbits of $\mathrm{SU}(2)$ in which the metric $g_{i j}$ is diagonal, that is $\left(X_{i}, X_{j}\right)=g_{i j}=-q_{i} \delta_{i j}$. The Einstein equations (1.1) after the time change

$$
d \tau=d t\left(q_{1} q_{2} q_{3}\right)^{-k / 2}
$$

are equivalent to a Hamiltonian system in the phase space $R^{6}\left(p_{i}, q_{i}\right)(i=1,2,3)$ :

$$
\dot{p}_{i}=-\frac{\partial H}{\partial q_{i}}, \quad \dot{q}_{i}=\frac{\partial H}{\partial p_{i}},
$$

where the Hamiltonian $H$ has the form

$$
H=\left(q_{1} q_{2} q_{3}\right)^{-(1-k) / 2}\left(2 \sum_{i<j}^{3} p_{i} q_{i} p_{j} q_{j}-\sum_{i=1}^{3} p_{i}^{2} q_{i}^{2}+\frac{1}{4}\left(2 \sum_{i<j}^{3} q_{i} q_{j}-\sum_{i=1}^{3} q_{i}^{2}\right)\right) .
$$

The value of $H$, which is conserved by virtue of (1.4), is connected with the energy density e by:

$$
H=(i+3 k) \varepsilon\left(q_{1} q_{2} q_{3}\right)^{(1+k) / 2} .
$$

The system (1.4) admits a group of scale transformations

$$
\varphi_{\lambda}: q_{i} \rightarrow \lambda_{i} q_{i}, \quad \varphi_{\lambda}: p_{i} \rightarrow p_{i}, \quad \varphi_{\lambda}: \tau \rightarrow \lambda^{(1-3 k) / 2} \cdot \tau
$$

and has the monotone scale-invariant function

$$
F=3 \frac{d}{d t}\left(q_{1} q_{2} q_{3}\right)^{1 / 6}=\frac{p_{1} q_{1}+p_{2} q_{2}+p_{3} q_{3}}{\left(q_{1} q_{2} q_{3}\right)^{1 / 3}}, \quad \frac{d F}{d \tau} \leqslant 0 .
$$

In what follows we examine the system (1.4) in a domain $S_{1} \subset R^{6}$, which is distinguished by the natural physical conditions of a positive space metric and energy density: $q_{i}>0, H \geqslant 0$. By a choice of the sign of time we have $F<0$ on the side of compression.

This is the programme for applying the ideas of the qualitative theory to this problem. Using the scale group $\varphi_{\lambda}$ (1.7), we can transform (1.4) from the domain $S_{1} \subset R^{6}$ into a system defined on a five-dimensional compact factor-manifold $S$ completed by a boundary $\Gamma$. The resulting system on $S$ can be extended continuously to $\Gamma$ and has a set of singular points, with integrable separatrices, lying only on $\Gamma$. We can now give a full description of the regimes of the behaviour of the metric for the nearly cosmological singularity $q_{1} q_{2} q_{3}=0$ by means of an approximation of the corresponding trajectory on $S$ by a sequence of singular point and separatrices past which the trajectory goes, approaching the boundary $\Gamma$ of $S$ around the cosmological singularity $q_{1} q_{2} q_{3}=0$. This programme was put into effect by the authors in [2] and [3] for $G=\mathrm{SU}(2)$ and for zero velocities of the material. 
II. We now proceed to the construction of the 5-dimensional compact manifold $S$. It is covered, by two local charts $W_{1}$ and $W_{2}$. In $W_{1}$ we introduce coordinates $\bar{s}_{i}, y_{i}$ :

$$
\bar{s}_{i}=p_{i} q_{i} / G, \quad y_{i}=q_{i} / G, \quad G=\left(q_{1}^{2}+q_{2}^{2}+q_{3}^{2}\right)^{1 / 2}
$$

In $W_{2}$ we introduce coordinates $s_{i}, y_{i}, w$ :

$$
\left\{\begin{array}{l}
s_{i}=\bar{s}_{i} /\left(\bar{s}_{1}^{2}+\bar{s}_{2}^{2}+\bar{s}_{3}^{2}\right)=p_{i} q_{i} / P \\
w=\left(\bar{s}_{1}^{2}+\bar{s}_{2}^{2}+\bar{s}_{3}^{2}\right)^{-1}=G^{2} / P^{2} \\
P=\left(p_{1}^{2} q_{1}^{2}+p_{2}^{2} q_{2}^{2}+p_{3}^{2} q_{3}^{2}\right)^{1 / 2} .
\end{array}\right.
$$

Here $s_{i}$ and $y_{i}$ satisfy two relations:

$$
s_{1}^{2}+s_{2}^{2}+s_{3}^{2}=1, \quad y_{1}^{2}+y_{2}^{2}+y_{3}^{2}=1 .
$$

The compact manifold $S$ with boundary $\Gamma$ in the coordinate domains $W_{1}$ and $W_{2}$ is distinguished by the conditions

$$
y_{i} \geqslant 0, \quad w \geqslant 0, \quad H_{1} \geqslant 0, \quad \bar{s}_{1}+\bar{s}_{2}+\bar{s}_{3} \leqslant 0
$$

where

$$
H_{1}=H\left(q_{1} q_{2} q_{3}\right)^{(1-k) / 2} / P^{2}=2 \sum_{i<j}^{3} s_{i} s_{j}-1+\frac{1}{4} w\left(2 \sum_{i<j}^{3} y_{i} y_{j}-1\right) .
$$

In accordance with (1.12) $y_{1}, y_{2}, y_{3}$ range over the 'triangle' $\Delta: y_{i} \geqslant 0$ on the unit sphere $S^{2}: y_{1}^{2}+y_{2}^{2}+y_{3}^{2}=1$. Let $S^{1}$ be the circle inscribed in $\Delta ; S^{1}$ is given on $S^{2}$ by the equations $y_{1} y_{2}+y_{2} y_{3}+y_{3} y_{1}=\frac{1}{2}$. If the point $\left(y_{1}, y_{2}, y_{3}\right)$ lies outside the circle $S^{1}$, then the condition $H_{1} \geqslant 0$ cuts out a compact set in the coordinates $s_{1}, s_{2}, s_{3}$, and $w$. If, however, $\left(y_{1}, y_{2}, y_{3}\right)$ lies on $S^{1}$ or inside, then the set $H_{1} \geqslant 0$ is non-compact in the coordinate $w: 0 \leqslant w \leqslant \infty$.

The boundary $\Gamma$ of $S$ is not smooth: $\Gamma$ consists of six components (boundaries) defined by the conditions

$$
\begin{array}{ll}
\Gamma_{0}: H_{1}=0, & \Gamma_{i}: y_{i}=0 \quad(i=1,2,3), \\
\Gamma_{w}: w=0, & \Gamma_{m}: \bar{s}_{1}+\bar{s}_{2}+\bar{s}_{3}=0 .
\end{array}
$$

The boundary components $\Gamma_{0}, \Gamma_{m}$ lie in the "physical" domain of $S$, that is, to these components there correspond non-singular states of the metric, whereas the boundary components $\Gamma_{1}, \Gamma_{2}, \Gamma_{3}$, and $\Gamma_{w}$ are compactifications of the physical domain $S$ with degenerate states of the metric and correspond to the cosmological singularity $q_{1} q_{2} q_{3}=0$.

The intersections of pairs of the various components form the corners of $\Gamma$.

It is convenient to use the coordinates (1.9) to study the behaviour of the system (1.4) in the neighbourhood of the maximal expanse, where $p_{1} q_{1}+p_{2} q_{2}+p_{3} q_{3}=0$, and the coordinates (1.10) in the neighbourhood of the cosmological singularity.

III. The system (1.4) in the coordinates (1.10) and the time $\tau_{1}$ :

$$
d \tau_{1} / d t=1 / 2\left(p_{1}^{2} q_{1}^{2}+p_{2}^{2} q_{2}^{2}+p_{3}^{2} q_{3}^{2}\right)^{1 / 2}\left(q_{1} q_{2} q_{3}\right)^{-1 / 2}>0
$$


has the form

$$
\left\{\begin{array}{l}
\dot{s}_{i}=w\left(-y_{i}\left(y_{1}+y_{2}+y_{3}-2 y_{i}\right)+s_{i} Z_{1}\right)+(1-k)\left(1-s_{i} Z_{2}\right) H_{1}, \\
\dot{w}=2 w\left[Z_{2}\left(4-(1-k) H_{1}\right)-8 Z_{3}+w Z_{1}\right] \\
\dot{y}_{i}=8 y_{i}\left(Z_{3}-s_{i}\right), \quad \dot{G}=4 G\left(Z_{2}-2 Z_{3}\right), \\
Z_{1}=\sum_{k=1}^{3} s_{k} y_{k}\left(y_{1}+y_{2}+y_{3}-2 y_{k}\right) \\
Z_{3}=\sum_{k=1}^{3} s_{k} y_{k}^{2}, \quad Z_{2}=s_{1}+s_{2}+s_{3} .
\end{array}\right\} .
$$

The system (1.14) extends continuously to $\Gamma$, and the boundary components $\Gamma_{0}$, $\Gamma_{1}, \Gamma_{2}, \Gamma_{3}, \Gamma_{w}$ are invariant manifolds of this system, and on $\Gamma_{m}$ the field (1.14) is directed towards $S$.

The monotone function $F(1.8)$ in the coordinates (1.10) has the form

$$
F=\frac{s_{1}+s_{2}+s_{3}}{w^{1 / 2}\left(y_{1} y_{2} y_{3}\right)^{1 / 3}}, \quad \frac{d F}{d \tau_{1}} \leqslant 0 .
$$

Since $F$ is monotone, it follows that if the time is directed towards the compression side, that is, $s_{1}+s_{2}+s_{3}<0$, then all the trajectories of (1.4) approximate to the boundary components $\Gamma_{1}, \Gamma_{2}, \Gamma_{3}$, and $\Gamma_{w}$, near which $F \rightarrow-\infty$. In particular, all singular points of (1.14) lie on $\Gamma$.

IV. The complete set of singular points of (1.14), consisting of sets of six types $\Phi_{L K}, N_{i}, T_{i}, A_{i}, B_{i},(\psi, i)$ is illustrated in Fig. 1.

1) The set $\Phi_{L K}$ lies on the boundary component $\Gamma_{w}$, is two-dimensional, and is defined by the conditions: $w=0, s_{i}=-1 / \sqrt{3}, y_{1}, y_{2}, y_{3}$ range over $\Delta$.

Figure 1. General disposition of singular points of a dynamical system of a model of type IX after accounting for scale-invariance and augmentation of the physical domain of the boundary. 
2) Three isolated singular points $N_{i}$ lie at the corners $\Gamma_{j} \cap \Gamma_{k}$ of the boundary and have the coordinates

$$
\begin{aligned}
s_{i} & =-2^{1 / 2}(3+k) u \\
s_{j} & =s_{k}=-(5-k) 2^{-1 / 2} u \\
w & =8(1+3 k)(1-k) u^{2} \\
u & =\left(43+2 k+3 k^{2}\right)^{-1 / 2} .
\end{aligned}
$$

3) Three segments of singular points $T_{i}$ lie on the boundary components $\Gamma_{i}$ and have the coordinates $y_{i}=0, y_{j}=y_{k}=2^{-1 / 2}, s_{i}=0, s_{j}=s_{k}=-2^{-1 / 2} ; w \geqslant 0$ is arbitrary. One end of $T_{i}$ has the coordinate $w=0$ and the other end, $T_{i}^{0}(w=\infty)$, in the local chart $W_{1}(1.9)$ has the coordinates $\bar{s}_{1}=\bar{s}_{2}=\bar{s}_{3}=0$, that is, it lies on the intersection $\Gamma_{1} \cap \Gamma_{0} \cap \Gamma_{m}$.

4) Three segments of singular points $A_{i}$ and three segments $B_{i}$ lie on the intersection of the boundary components $\Gamma_{i} \cap \Gamma_{w} \cap \Gamma_{0}$. At these singular points $y_{i}=0$, $y^{2}+y_{k}^{2}=1, w=0$. On $A_{i}$ we have $s_{i}=-2^{3 / 2} / 3, s_{j}=s_{k}=-2^{-1 / 2} / 3$. On $B_{i}$ we have $s_{i}=0, s_{j}=s_{k}=-2^{-1 / 2}$.

5) Three circles of singular points $(\psi, i)$ lie at the corners of $\Gamma_{j} \cap \Gamma_{k} \cap \Gamma_{w} \cap \Gamma_{0}$ and we have the coordinates $y_{k}=\delta_{k l}, w=0$. The coordinates $s_{1}, s_{2}, s_{3}$ satisfy the equations $s_{1}^{2}+s_{2}^{2}+s_{3}^{2}=1, s_{1} s_{2}+s_{2} s_{3}+s_{3} s_{1}=\frac{1}{2}$.

The singular sets $\Phi_{L K}, N_{i}, T_{i}$ are non-degenerate (that is, for them the number of eigenvalues zero of (1.14) is equal to the dimension of these sets) and unstable. The separatrices entering these singular points from the physical domain determine the asymptotic behaviour, in powers of $t$, of the metric under the contraction of space $(t \rightarrow 0)$ :

$$
\begin{gathered}
q_{i} \cong C_{i} t^{4 / 3(1-k)}, \\
q_{i} \cong C_{i} t^{(1-k) /(1+k)}, \quad q_{j} \cong C_{j} t^{(3+k) / 2(1+k)}, \quad q_{k} \cong C_{k} t^{(3+k) / 2(1+k)}, \\
q_{i} \cong C t^{2}, \quad q_{j} \cong q_{k} \cong C_{1} .
\end{gathered}
$$

The asymptotic forms (1.16) for $\left(\Phi_{L K}\right)$ generalize the Friedmann solution $q_{1} \equiv$ $q_{2} \equiv q_{3}$. They were first found by Lifshits and Khalatnikov [22]. The asymptotic forms (1.17) for $\left(N_{i}\right)$, like (1.16), occur only in occupied space $(H \neq 0)$ and were first found by one of the present authors [1]. The forms (1.18) for $\left(T_{i}\right)$ occur both in occupied and in empty space and were discovered by Taub [23].

The asymptotic forms (1.16)-(1.18) exhaust all the asymptotic expansions in powers of the metric of a model of type IX in the neighbourhood of a singularity. The singular segments $A_{i}$ and $B_{i}$ have extra zero eigenvalues; it can be shown that to these points there do not correspond any asymptotic forms in powers of $t$. The singular circles $(\psi, i)$ at the corners of $\Gamma$ are non-degenerate and unstable. All their separatrices lie on $\Gamma$, therefore, to them there do not correspond asymptotic forms in powers. The circles $(\psi, i)$, together with their separatrices, determine an approximation to the complex oscillating regime of the metric in the neighbourhood of the cosmological singularity, which was first discovered in [8].

$\mathrm{V}$. When the direction of time is towards the compression, all the trajectories of (1.14), by virtue of the presence of the monotone function $F(1.15)$, approximate to the boundary components $\Gamma_{1}, \Gamma_{2}, \Gamma_{3}$, and $\Gamma_{w}$. Along each trajectory, $F \rightarrow-\infty$. A trajectory of $(1.14)$, when it falls into a neighbourhood of the boundary $\Gamma$ defined 
by condition $|F| \gg 1$, begins to move along those trajectories of the system that lie on $\Gamma$. It can be shown that all trajectories of (1.14) on $\Gamma$ are separatrices of singular points and lead from one singular point to another. After finitely many transfers (never more than 3 ) along the separatrices of singular points $\Phi_{L K}, N_{i}, T_{i}, A_{i}$, and $B_{i}$, the trajectory falls into the neighbourhood of singular points $(\psi, i)$ and begins to travel along their separatrices. On the side of compression, all the separatrices leaving singular points $(\psi, i)$ go to singular points of the same kind $(\psi, k)$. Thus, there is an infinite sequence of separatrix transfers of the form

$$
\cdots \rightarrow\left(\psi_{1}, i_{1}\right) \rightarrow\left(\psi_{2}, i_{2}\right) \rightarrow\left(\psi_{3}, i_{3}\right) \rightarrow \cdots,
$$

leading to a "BLK regime". (See [3] for a more detailed description.)

VI. When the direction of time is towards expansion a trajectory of (1.14) beginning in a small neighbourhood of $F$ distinguished by the condition $F \gg 1$ moves to the "reverse side" along the separatrix on $\Gamma$ until it falls into the neighbourhood of singular points having separatrices that go into the physical domain $S$. When the trajectory moves from $\Gamma$ along the separatrices, the metric is approximated by the power regimes (1.16)-(1.18), which are therefore typical states of the metric at the early stage of expansion. The final transfers between singular points immediately preceding the departure of the trajectory from $\Gamma$ into the physical domain $S$ are shown in Fig. 2. Here the straight arrow $\rightarrow$ signifies a transfer along a separatrix; the number above the arrow indicates the dimension of this separatrix; the dots denote singular points of type $(\psi, i), A_{i}, B_{i}$, and the wavy arrow $\rightsquigarrow$ indicates a transition by continuity from the inclusion of the corners $\Phi_{i}^{\infty}$ and sides $\Phi_{i}^{0}$ in the triangle of singular points $\Phi_{L K}$.

FiguRE 2. The final paths in the evolution of the metric towards expansion before departure from the singularity.

The separatrix transfers shown in Fig. 2 give a complete list of all possible paths in the evolution of the metric of a homogeneous cosmological model of type IX in the neighbourhood of the cosmological singularity under expansion.

As further research has shown ([4], [24]-[26]), conclusions about the process of evolution of the universe in the early stages of expansion scarcely depend upon the type of the homogeneous model.

The programme for applying the methods of the qualitative theory to the remaining models (among them those accounting for the velocities of the material) was drawn up basically in papers by Bogoyavlenskii [4], [25], [26] and for the case of a model of type VII was completed by Persetskii. 


\section{$\S$ 2. Dynamics of a gravitating gas Ellipsoid}

I. The motion of a gravitating ideal gas filling a certain ellipsoid at a constant density is a special case of the motion of a continuous medium in which the velocities are linear functions of the coordinates. Such motions of a continuous medium have been studied in a large number of works of which the first were the classical investigations of Riemann, Dirichlet and Dedekind on the theory of figures of equilibrium of an ideal incompressible gravitating liquid (see the bibliography in [21] and [6]). In [28] and [29] numerical methods were used in discovering the oscillating nature of the adiabatic motion of a gravitating gas ellipsoid with the total energy of the gas $E<0$ and with the exponent of the adiabatic curve $\gamma<4 / 3$.

In this section we show, with the help of methods similar to the qualitative theory of homogeneous cosmological models (see $\S 1$ ), that for certain values of the parameters the oscillating regime can be approximated by a sequence of simpler motions of a gravitating dust ellipsoid, which has been studied in [19] and [30]. The oscillating regime, in contrast to the spherically symmetrical regime of compression of a gas to a point, is stable in the relevant class of gas motions. The approximation to the oscillating regime obtained here becomes exact as $E \rightarrow-\infty$ (strong compression) or as $\beta \rightarrow 0$ ( $\beta$ is a characteristic parameter of the problem).

II. The adiabatic motion of a gravitating ideal gas is known to be determined by the equations

$$
\rho \frac{d \mathrm{v}}{d t}=-\operatorname{grad} p-\rho \operatorname{grad} \Phi, \quad \frac{d \rho}{d t}=-\rho \operatorname{div} \mathrm{v} .
$$

Here $\rho$ is the gas density, $\mathrm{v}$ the velocity, $p$ the pressure, $\gamma>1$ the exponent of the adiabatic curve $\frac{d}{d t}\left(\rho^{\gamma} / p\right)=0$, and $\Phi$ the Newtonian potential created by the whole mass of gas. We consider a solution of the equations (2.1) satisfying the following conditions: the Euler coordinates $r_{i}$ are linear functions of the Lagrange coordinates $a_{k}$ :

$$
r_{i}=\sum_{k=1}^{3} F_{i k}(t) a_{k} \quad(i=1,2,3)
$$

(Therefore, the velocities $v_{i}=d r_{i} / d t$ are linear functions of the $r_{k}$.) The density $\rho$ and pressure $p$ of the gas for $a^{2}=a_{1}^{2}+a_{2}^{2}+a_{3}^{2} \leqslant 1$ are determined by the formulae

$$
\left\{\begin{array}{l}
\rho=\frac{3 M}{4 \pi} V^{-1}(F), \quad p=\alpha \frac{3 M}{4 \pi}(\gamma-1)\left(1-a^{2}\right) V^{-\gamma}(F), \\
V(F)=\operatorname{det}\left\|F_{i k}\right\| .
\end{array}\right.
$$

Here $\alpha$ and $M$ are constants; $M$ is the total mass of the gas. When $a^{2}>1$, we put $\rho=0, p=0$.

By virtue of (2.3), gas at a constant density fills the ellipsoid that is obtained from the unit sphere $a^{2} \leqslant 1$ by the mapping $F_{i k} ; p$ is maximal at the centre of the ellipsoid and equal to 0 on its surface. 
Let $d_{1}, d_{2}, d_{3}$ be the semi-axes of the ellipsoid; it is known (see [31]), that the Newtonian potential $\Phi$ at a point $\left(x_{1}, x_{2}, x_{3}\right)$ within the ellipsoid has the form

$$
\begin{aligned}
& \Phi\left(x_{1}, x_{2}, x_{3}\right)= \\
= & \frac{3}{4} G M \int_{0}^{\infty}\left(\frac{x_{1}^{2}}{d_{1}^{2}+s}+\frac{x_{2}^{2}}{d_{2}^{2}+s}+\frac{x_{3}^{2}}{d_{3}^{2}+s}\right)\left(\left(d_{1}^{2}+s\right)\left(d_{2}^{2}+s\right)\left(d_{3}^{2}+s\right)\right)^{-1 / 2} d s
\end{aligned}
$$

Here $G$ is the gravitational constant.

Following the method of [16] and [17] and using (2.2)-(2.4) we can show that for the motions in question the equations (2.1) are equivalent to the following Lagrange system, which is defined in the space of three-dimensional matrices:

$$
\left\{\begin{array}{l}
\frac{d^{2} F_{i k}}{d t^{2}}=-\alpha \frac{\partial V^{1-\gamma}(F)}{\partial F_{i k}}+\frac{3 G M}{8} \frac{\partial U(F)}{\partial F_{i k}} \\
U(F)=\int_{0}^{\infty}\left(\left(d_{1}^{2}+s\right)\left(d_{2}^{2}+s\right)\left(d_{3}^{2}+s\right)\right)^{-1 / 2} d s
\end{array}\right.
$$

Thus, the study of the motions of a gravitating ideal gas described above is equivalent to that of the motions of a material point in the nine-dimensional space of the matrices $F_{i k}$ in a field with the potential given by (2.5). We note that the system (2.5) depends on a single characteristic parameter $\beta=8 \alpha / 3 G M$, which cannot be eliminated by a time change.

Remark. Since $d_{1}^{2}, d_{2}^{2}, d_{3}^{2}$ are eigenvalues of the matrix $F \cdot F^{t}$, the integrand in (2.5) can be expressed in the following way in terms of the components of $F_{i k}$ :

$$
\begin{aligned}
& \left(d_{1}^{2}+s\right)\left(d_{2}^{2}+s\right)\left(d_{3}^{2}+s\right)= \\
& \quad=\operatorname{det}\left(F \cdot F^{t}\right)+\frac{1}{2} s\left[\left(\operatorname{Tr}\left(F \cdot F^{t}\right)\right)^{2}-\operatorname{Tr}\left(F \cdot F^{t} \cdot F \cdot F^{t}\right)\right]+s^{2} \operatorname{Tr}\left(F \cdot F^{t}\right)+s^{3} .
\end{aligned}
$$

Here $F^{t}$ is a transposed matrix and $\operatorname{Tr}(X)$ is the trace of a matrix $X$.

III. In the phase space with the coordinates $P_{i}=\dot{F}_{j k}, q_{i}=F_{j k}(i=1, \ldots, 9$; $j=1, \ldots, 9)$, the energy $E$ (the Hamiltonian) has the form $(n=9)$

$$
E=\frac{1}{2}\left(P_{1}^{2}+\cdots+P_{n}^{2}\right)+\alpha V^{1-\gamma}\left(q_{i}\right)-\frac{3}{8} G M U\left(q_{i}\right) .
$$

We introduce new coordinates in the phase space:

$$
\begin{gathered}
p_{i}=P_{i}\left(\alpha V^{1-\gamma}\left(q_{i}\right)+\frac{3}{8} \operatorname{GMU}\left(q_{i}\right)\right)^{-1 / 2}, \\
\left\{\begin{array}{l}
u=U\left(q_{i}\right)\left(\beta \cdot V^{1-\gamma}+U\left(q_{i}\right)\right)^{-1}, \\
y_{i}=q_{i}\left(q_{1}^{2}+\cdots+q_{n}^{2}\right)^{-1 / 2} .
\end{array}\right.
\end{gathered}
$$

The $y_{i}$ range over the unit sphere $S^{n-1}: y_{1}^{2}+\cdots+y_{n}^{2}=1$; the $p_{i}$ over the whole Euclidean space $R^{n}$, and the $u$ over the interval $0<u<1$. Note that when the $u$ and $y_{i}$ become dependent for $\gamma=4 / 3$; from now on we put $\gamma<4 / 3$.

The Lagrangian system (2.2) in the coordinates (2.6) and the time

$$
\frac{d \tau_{1}}{d t}=\frac{\left(\alpha V^{1-\gamma}\left(q_{i}\right)+\frac{3}{8} G M U\left(q_{i}\right)\right)^{1 / 2}}{\left(q_{1}^{2}+\cdots+q_{n}^{2}\right)^{1 / 2} V(y)}
$$


has the following form:

$$
\left\{\begin{array}{l}
\dot{p}_{i}=(1-\gamma)(1-u)\left(-\frac{\partial V}{\partial y_{i}}-\frac{1}{2} p_{i} W_{1}\right)+u \frac{V(y)}{U(y)}\left(\frac{\partial U}{\partial y_{i}}-\frac{1}{2} p_{i} W_{2}\right) \\
\dot{y}_{i}=V(y)\left(p_{i}-y_{i}\left(p_{k} y_{k}\right)\right) \\
\dot{u}=u(1-u)\left(\frac{V(y)}{U(y)} W_{2}-(1-\gamma) W_{1}\right) \\
W_{1}=\frac{\partial V}{\partial y_{k}} p_{k}, \quad W_{2}=\frac{\partial U}{\partial y_{k}} p_{k}
\end{array}\right.
$$

(summation takes place over repeated indices $k$ ). Below we examine the system (2.9) for $E<0$. The energy $E$ in the coordinates (2.7) has the form

$$
\left\{\begin{aligned}
E=\frac{3}{8} G M \beta^{m} V^{m(1-\gamma)}(y)(U(y) / u)^{3 m(1-\gamma)} \times \\
\quad \times(1-u)^{-m}\left(\frac{1}{2}\left(p_{1}^{2}+\cdots+p_{n}^{2}\right)+1-2 u\right), \\
m=1 /(4-3 \gamma) .
\end{aligned}\right.
$$

Hence it follows that the domain $E \leqslant 0$ (or $p_{1}^{2}+\cdots+p_{n}^{2} \leqslant 4 u-2$ ), is bounded.

IV. The domain $S_{1}$ on which the system (2.9) is defined is given in the coordinates (2.7) by the conditions $0<u<1, w>0, V\left(y_{i}\right)>0$. (To the points of the surface $V\left(y_{i}\right)=0$ there corresponds in accordance with (1.3) a physical singularity of the solution.) To $S_{1}$ we add the boundary $\Gamma$, which consists of four components defined by the following conditions: $\Gamma_{0}: u=0 ; \Gamma_{1}: u=1 ; \Gamma_{2}: V\left(y_{i}\right)=0 ; \Gamma_{w}: w=0$. We denote by $S$ the manifold obtained as the result of this addition of the boundary. (On $S$ we have $0 \leqslant u \leqslant 1, w \geqslant 0, V\left(y_{i}\right) \geqslant 0$.) It is obvious that the system (2.9) can be extended continuously to the boundary components $\Gamma_{0}, \Gamma_{1}, \Gamma_{w}$. Using simple properties of the potential $U\left(q_{i}\right)$ (see [31]) we can show that $V(y) / U(y)$. $\partial U / \partial y_{i} \rightarrow 0$, as $V(y)=\operatorname{det}\left\|Y_{i k}\right\| \rightarrow 0$. Therefore, on the boundary component $\Gamma_{2}$ we supplement these expressions by zero, their limiting value. As a result of this process the system (2.9) is extended continuously to the boundary component $\Gamma_{2}$.

It is not difficult to verify that all the components of $\Gamma$ and their intersections are invariant submanifolds of the dynamical system (2.9) in $S$. The system so defined on the component $\Gamma_{0}(u=0)$, is identical with the system describing the motion of a gaseous non-gravitating ellipsoid, and that defined on $\Gamma_{1}(u=1)$ is identical with the system describing the motion of a gravitating dust-ellipsoid.

All the singular points of (2.9) on $S$ lie on $\Gamma$ when $E \leqslant 0$ and $\gamma<4 / 3$ and form four sets: $K, \Psi_{+}, \Psi_{-}$, and $L$.

1) The singular points $K(u=1, V(y)=0)$ are the intersection of the invariant submanifolds $\Gamma_{1}(u=1)$ and $\Gamma_{2}(V(y)=0)$. These singular points are nondegenerate (when $W_{1} \neq 0$ ) and unstable, and have two non-zero eigenvalues (the directions of the corresponding eigenvectors are shown in brackets)

$$
\begin{cases}\lambda_{1}=(1-\gamma) W_{1} & (\text { variables } u), \\ \lambda_{2}=W_{1} & \left(\text { variables } y_{i}\right)\end{cases}
$$

The remaining $2 n-2$ zero eigenvalues correspond to directions touching the manifolds $K$. Since $\gamma>1$, the signs of the eigenvalues $\lambda_{1}$ and $\lambda_{2}$ are opposite, that is the points are of the saddle type. It is convenient to divide $K$ into two parts 
$K_{+}\left(W_{1}>0\right)$ and $K_{-}\left(W_{1}<0\right)$. Each singular point of $K_{+}$has one entering separatrix going along $\Gamma_{2}$, and one departing separatrix going along $\Gamma_{1}$, and vice versa for the points of $K_{-}$.

2) The singular points $\Phi_{\varepsilon}\left(u=1, p_{i}=\varepsilon 2^{1 / 2} y_{i}, \varepsilon= \pm 1, y_{i}=Y_{j k}=3^{-1 / 2} Q_{j k}\right.$; $Q_{j k}$ is an orthogonal matrix). The calculation of the eigenvalues of (2.9) at the singular points $\Phi_{\varepsilon}$ shows that. they are non-degenerate and unstable. Here each point of the three-dimensional manifold $\Phi_{-}$has a four-dimensional entering separatrix formed by diagonal solutions (with a spherically symmetric compressive character) generalizing the exact spherically symmetrical solutions, and an eleven-dimensional entering separatrix (lying on $\Gamma_{2}$ at zero-level of energy). In view of this, the spherically symmetric compression is unstable. The properties of the singular points $\Phi_{+}$ are identical with those of $\Phi_{-}$, but with the opposite directions of time.

3) The degenerate singular points $L: V(y)=0, \partial V / \partial y_{i}=0 ; p_{i}, u$ are arbitrary. At these singular points $Y_{j k}$ is doubly degenerate.

Thus, when $E \leqslant 0$ and $\gamma<4 / 3$, there are no stable singular points in the system (2.9); this is one of the reasons for the existence of an oscillating regime.

V. As was mentioned above, the separatrices of the singular points $K_{+}$and $K_{-}$ lie on the invariant manifolds $\Gamma_{1}$ and $\Gamma_{2}$. Let us examine the system (2.9) on these manifolds.

1) The system $(2.9)$ on $\Gamma_{1}(u=1)$ describes the motion of a gravitating dustellipsoid. This form of motion is studied in [19], where it is shown, in particular, that along each solution with negative energy $E$ the volume $V(F)=\operatorname{det}\left\|F_{j k}\right\|$ of the ellipsoid vanishes of order 2 , that is, the expansion from the compressed state is changed into contraction. For almost all solutions the ellipsoid in the initial and final stage is compressed into a disk, that is, $d_{1}=0, d_{2} \neq 0, d_{3} \neq 0$. In the coordinates $(2.7)$ this result means that almost all trajectories of the system $(2.9)$ on $\Gamma_{1}$ for $E<0$ have their beginning and their end in the set of singular points $K$ $(V(y)=0, u=1)$, or for almost every singular point of $K_{+}$the separatrix departing from it goes to a certain singular point of $K_{-}$.

2) The system $(2.9)$ on $\Gamma_{2}(V(y)=0)$ can be integrated explicitly. The trajectories of this system in time $\tau$ are determined by the expressions $d \tau=2^{1 / 2}(\gamma-1) \times$ $(1-u)\left|\operatorname{grad} V\left(y_{i}^{0}\right)\right| d \tau_{1}$, has the form

$$
\left\{\begin{array}{l}
y_{i}=y_{i}^{0}, \quad p_{i}=\left(2^{1 / 2} s_{i}\left(\sin \tau-\sin \tau_{0}\right)+p_{i}^{0} \cos \tau_{0}\right) / \cos \tau, \\
u=\cos ^{2} \tau_{0} / \cos ^{2} \tau .
\end{array}\right.
$$

where $y_{i}^{0}, s_{i}=\operatorname{grad} V\left(y_{i}^{0}\right) /\left|\operatorname{grad} V\left(y_{i}^{0}\right)\right|, \tau_{0}, p_{i}^{0}$-are constants, where $V\left(y_{i}^{0}\right)=0$, $p_{1}^{0} s_{1}+\cdots+p_{n}^{0}=2^{1 / 2} \tan \tau_{0}<0,\left|\tau_{0}\right|<\pi / 2, p_{1} s_{1}+\cdots+p_{n} s_{n}=2^{1 / 2} \tan \tau$. The trajectory (2.12) is defined for $\tau_{0} \leqslant \tau \leqslant-\tau_{0}$ and goes from the singular point $\left(p_{i}^{0}, y_{i}^{0}, u=1\right)$ in $K_{-}$to the singular point $\left(p_{i}^{1}=p_{i}\left(-\tau_{0}\right), y_{i}^{0}, u=1\right)$ in $K_{+}$. (Consequently, all trajectories (2.12) are separatrices of the singular points of $K_{+}$ and $\left.K_{-}.\right)$It is easy to see that the end-point of the trajectory $(2.12)\left(p_{i}^{1}=p_{i}\left(-\tau_{0}\right)\right)$ is reached from the initial point $\left(p_{i}^{0}\right)$ along the path reflected in the plane tangential to the surface $V\left(y_{i}\right)=0$ at $\left(y_{i}^{0}\right)$.

The results obtained lead to the following separatrix diagram:

$$
\cdots \rightarrow K_{+} \stackrel{\mathrm{I}}{\rightarrow} K_{-} \stackrel{\mathrm{II}}{\rightarrow} K_{+} \stackrel{\mathrm{I}}{\rightarrow} \cdots
$$

Here the mappings marked by arrows indicate the passage along a separatrix from its initial to its final point. The mappings I and II are effected by the separatrices 
going along $\Gamma_{1}$ and $\Gamma_{2}$, respectively. The separatrix transition between the sets $K_{+}, K_{-}$and $\Phi_{\varepsilon}, L$ are not shown in diagram (2.3) because for almost all singular points of $K_{+}$and $K_{-}$the whole infinite sequence of mappings (2.13) does not go outside $K_{+}$and $K_{-}$.

VI. The infinite sequence of separatrices defined in (2.13) is an approximation to the trajectories of the system (2.9) for large negative energy $E$, and also for $\beta=8 \alpha / 3 G M \rightarrow 0$. For the function $E$ (see (2.10)) is bounded below everywhere on $S$ except for the boundary components $\Gamma_{1}(u=1)$ and $\Gamma_{2}(V(y)=0)$, where $E \rightarrow$ $-\infty$. Therefore, the trajectories of (2.9) with large negative energy $E$ remain all the time in a small neighbourhood of $\Gamma_{1}$ and $\Gamma_{2}$ (this is also true for any $E<0$, but $\beta \rightarrow 0$ see (2.7), the definition of the coordinate $u$ ). Consequently, these trajectories follow the trajectories of $(2.9)$ on $\Gamma_{1}$ and $\Gamma_{2}$, that is, the general trajectory of $(2.9)$ follows the sequence of separatrices of the singular points of $K_{+}$and $K_{-}$.

This approximation to the trajectories of (2.9) by the sequence of separatrices (2.13) proves that the general motion of a gravitating gas ellipsoid with large negative energy $E$, or with small parameter $\beta$, has a pulsating, oscillatory character. For according to (2.13) the trajectory periodically appears in the neighbourhood of singular points of $K_{+}, K_{-}$, where $\operatorname{det}\left\|Y_{j k}\right\|=V\left(y_{i}\right)=0$; that is, the ellipsoid is periodically compressed into a disc. Moreover, from the equations

$$
\frac{d V\left(q_{i}\right)}{d \tau_{1}}=V\left(q_{i}\right) W_{1}
$$

it follows that the volume $\operatorname{det}\left\|F_{j k}\right\|=V\left(q_{i}\right)$ of the ellipsoid attains a maximum for motion of the trajectory of (2.9) along the separatrix transition I, and a minimum for motion of the trajectory along the separatrix transition II (see (2.13)). Therefore, the variation in the density $\mathrm{p}$ (2.3) of the gas filling the ellipsoid also has oscillatory character. By (2.8) and (2.10) the period of each pulsation of the ellipsoid becomes arbitrarily small as $E \rightarrow-\infty$.

The pulsating motion of the ellipsoid so described, as $E \rightarrow-\infty$, arises in a state of strong compression in so far as (see (2.7)) the quantity

$$
d_{1}^{2}+d_{2}^{2}+d_{3}^{2}=q_{1}^{2}+\cdots+q_{9}^{2}=\beta^{-2 m}(U(y)(1-u) / u)^{2 m} V(y)^{2 m(\gamma-1)} .
$$

$m=1 /(4-3 \gamma)$, tends to zero as $E \rightarrow-\infty$ (that is, for $V(y) \rightarrow 0$ or $u \rightarrow 1$ ). We note, however, that in the presence of rotation of the gas the ellipsoid cannot be compressed beyond a definite dimension. As is known [17], Lagrangian systems of type (2.5) have first integrals $J$ and $K$ (connected with the full moment of the amount of motion of the gas and with vorticity):

$$
J=F \circ \dot{F}^{t}-\dot{F} \circ F^{t}, \quad K=F^{t} \circ \dot{F}-\dot{F}^{t} \circ F .
$$

It can be shown that when $E \leqslant 0, L \neq 0(L=\max \{|J|,|K|\})$, the following inequalities hold:

$$
A^{2} B^{2 /(\gamma-1)} / \ln B<d_{i}<(\beta(\gamma-1))^{1 /(3 \gamma-4)},
$$

where

$$
B=D / 2 \log D, \quad D=A(\beta(\gamma-1))^{1 / 2(4-3 \gamma)}, \quad A=\frac{2}{3} L((\gamma-1) / G M)^{1 / 2} .
$$

The separatrix approximation (2.13) means that asymptotically the motion of the trajectories of the system (2.9) in the coordinates $y_{i}$, as $E \rightarrow-\infty$ or $\beta \rightarrow 0$, proceeds in the following way. 
1) In the domain $V\left(y_{i}\right)<0$ the motion occurs along the trajectory corresponding to the gravitating dust-ellipsoid. In general, this trajectory is cut by the surface $V\left(y_{i}\right)=0$ at a certain point $y_{i}^{0}$ (transition I).

2) At the point of intersection the trajectory is elastically reflected from the surface $V\left(y_{i}\right)=0$ (transition II, see (2.13)).

3) Then motion again occurs along the trajectory corresponding to the gravitating ellipsoid up to the next intersection with the surface $V\left(y_{i}\right)=0$, and so on.

Thus, we can say that many-dimensional billiards in the domain $\operatorname{det}\left\|Y_{j k}\right\|=$ $V\left(y_{i}\right) \geqslant 0$ on the eight-dimensional sphere $S^{8}\left(\operatorname{Tr}\left(Y \circ Y^{t}\right)=1\right)$ with the elastically reflecting surface det $\left\|Y_{j k}\right\|=0$ provides a model for the oscillating regime of the motion of a gravitating gas ellipsoid. Between collisions with the boundary, the point moves along trajectories describing the motion of a gravitating dust-ellipsoid. The presence of hydrodynamic pressure is shown in the property of elastic reflection of the trajectory from the boundary $\operatorname{det}\left\|Y_{j k}\right\|=0$.

Note. Owing to the fact that the system (2.9), on the manifolds $\Gamma_{1}$ and $\Gamma_{2}$, does not depend on $\gamma$ when $\gamma>4 / 3$, there also exists an oscillating regime of motion of an ellipsoid approximated by the separatrix diagram (2.13). However, the physical content of this oscillating regime is quite different. For when $\gamma>4 / 3, E$ is close to zero according to (2.10) in the neighbourhood of $\Gamma_{1}$ and $\Gamma_{2}$, and the quantity $d_{1}^{2}+d_{2}^{2}+d_{3}^{2}$ is unbounded by $(2.15)$. Therefore, in the oscillating regime when $\gamma>4 / 3, E<0$, the ellipsoid has small energy and the gas is in a rarified state, and the period of each oscillation as $E \rightarrow 0$ becomes arbitrarily large.

\section{APPENDIX}

\section{Oscillating Regimes in Systems with Exponential Potential}

\section{O. I. Bogoyavlenskii}

There is an important class of Hamiltonian systems in which complicated nonlinear oscillating regimes can be studied by qualitative methods. These systems have the form

$$
\left\{\begin{array}{l}
\dot{p}_{i}=-\partial H / \partial q_{i}, \quad \dot{q}_{i}=\partial H / \partial p_{i}, \\
H=\frac{1}{2} \sum_{i, j}^{n} a_{i j} p_{i} p_{j}+\sum_{k, m}^{n+1} b_{k m} \exp \left(\{\alpha, q\}+\left\{\alpha_{m}, q\right\}\right) .
\end{array}\right.
$$

Here $\alpha_{1}, \ldots, \alpha_{n+1}$ are vectors in $R^{n}$ having the coordinates $\alpha_{k}=\left(d_{k_{1}}, \ldots, d_{k_{n}}\right)$, and $q$ is the vector $\left(q_{1}, \ldots, q_{n}\right)$. In $R^{n}$ inner products are given by

$$
(x, y)=\sum_{i, j}^{n} a_{i j} x_{i} y_{j}, \quad\{x, y\}=\sum_{i=1}^{n} x_{i} y_{i}
$$

Subject to $\alpha_{1}+\cdots+\alpha_{n+1}=0$ all the systems (3.1) under the mapping

$$
Q_{k}=\exp \left(\alpha_{k}, q\right) \quad(k=1, \ldots, n+1)
$$

become systems of hydrodynamical kind in the terminology of [32]. Particular cases of systems of the form (3.1) are given by a periodic Toda chain with the Hamiltonian

$$
H=\frac{1}{2} \sum_{i=1}^{n+1} p_{i}^{2}+\sum_{i=1}^{n} \exp \left(q_{i}-q_{i+1}\right)+\exp \left(q_{n+1}-q_{1}\right)
$$


and by periodic permutations of a Toda chain, and also as a homogeneous cosmological model of type IX (on the level $H=0$ ) with the Hamiltonian

$$
H=2 \sum_{i<j}^{3} p_{i} p_{j}-\sum_{i=1}^{3} p_{i}^{2}+2 \sum_{i<j}^{3} \exp \left(q_{i}-q_{j}\right)-\sum_{i=1}^{3} \exp \left(2 q_{i}\right) .
$$

In systems (3.1) there arises an infinite oscillating regime if the vectors $\alpha_{1}, \ldots$, $\alpha_{n+1}$ and the quadratic forms in $a_{i j}, b_{k m}$ satisfy the following conditions $\mathrm{A}$ and B:

A. $\max _{k}\left(\alpha_{k}, p\right)>0$ for each vector $p$ in $R^{n}$;

B. $\left(\alpha_{k} \alpha_{k}\right), \beta_{k k}>0$ for each $k$.

The oscillating regime in case (3.3) goes over into the standard oscillating regime in relativistic cosmology (see $\S 1$ ).

The separatrix approximation to the oscillating regime in (3.1) is connected with the Coxeter group $G$ generated by the reflections $\tau_{k}(k=1, \ldots, n+1) ; \tau_{k}(p)=$ $p-\frac{2\left(\alpha_{k}, p\right)}{\left(\alpha_{k}, \alpha_{k}\right)} \alpha_{k}$. For a positive definite metric $a_{i j}$ this approximation is applicable when $H \gg 1$.

For the periodic Toda chain (3.2), $G$ is a permutation group $U_{n+1}$. The property of $G$ being finite distinguishes an integrable Toda chain (3.2) among its general nonintegrable perturbations (3.1). In general, $G$ is infinite and the oscillating regime in (3.1) has stochastic qualities.

There are exceptional Hamiltonian systems of the form (3.1) connected with the simple Lie algebras $\mathfrak{G}$, which admit, like the Toda chain, a presentation in the form $L-A$ pairs, having therefore a large set of first integrals (in these systems $G$ is finite, and stochastization is impossible). Let $\omega_{1}, \ldots, \omega_{n}$ be the basis of simple roots of the Lie algebra $\mathfrak{G}$, let $\Omega=k_{1} \omega_{1}+\cdots+k_{n} \omega_{n}$ be a maximal root, and let the scalar product $(x, y)$ in $\mathfrak{G}$ be defined by the Killing-Cartan form. The Hamiltonian system in the $2 n$-dimensional phase space $p_{i}, q_{i}$ with the Hamiltonian

$$
H=\frac{1}{2} \sum_{i, j}^{n}\left(\omega_{i}, \omega_{j}\right) p_{i} p_{j}+\sum_{i=1}^{n} \exp \left(q_{i}\right)+b \exp \left(-k_{1} q_{1}-\cdots-k_{n} q_{n}\right)
$$

admits a presentation in the form of $L-A$ pairs, where the operators $L$ and $A$ belong to a certain faithful linear presentation of Lie algebra $\mathfrak{G}$. For a simple Lie algebra of type $A_{n}$ the Hamiltonian (3.4) defines a periodic Toda chain (3.2) (with zero total impulse $p_{1}+\cdots+p_{n+1}=0$ ). For algebras of the types $B_{n}, C_{n}, D_{n}$ the Hamiltonian systems (3.4) have the form of a Toda chain with special boundary conditions.

\section{REFERENCES}

[1] S. P. Novikov, On certain properties of cosmological models, Zh. Eksper. Teoret. Fiz. 62 (1972), 1977-1989.

= Soviet Physics JETP 35, 1031-1037.

[2] O. I. Bogoyavlenskii and S. P. Novikov, Properties of cosmological models of Bianchi IX type from the point of view of the qualitative theory of differential equations, Zh. Eksper. Teoret. Fiz. 64 (1973), 1475-1494.

= Soviet Physics JETP 37, 747-755.

[3] O. I. Bogoyavlenskii and S. P. Novikov, The qualitative theory of homogeneous cosmological models, Trudy Petrovskii Sem. 1 (1975).

[4] O. I. Bogoyavlenskii, On some properties of a cosmological model of type IX with motion of the material. Zh. Eksper. Teoret. Fiz. 70 (1976), 361-373. 
[5] O. I. Bogoyavlenskii, The oscillating regime of the expansion of a gas cloud in a vacuum, Letters to the Astronom. Zh. 1:9 (1975), 22-26.

[6] O. I. Bogoyavlenskii, The dynamics of a gravitating gas ellipsoid, Prikl. Mat. Mekh. 40 (1976), $270-280$.

[7] Ya. B. Zel'dovich and I. D. Novikov. Stroenie $i$ evotyutsiya Vselennoi (The structure and evolution of the universe), Nauka, Moscow 1975.

[8] V. A. Belinskii, E. M. Lifshits, and I. M. Khalatnikov: The oscillating regime of approximation to a singular point in relativistic cosmology, Uspekhi Fiz. Nauk 102 (1970), 463-500. = Soviet Phys. Uspekhi 13, 745-765.

[9] C. W. Misner, Mixmaster Universe, Phys. Rev. Lett. 22 (1969), 1071-1074.

[10] C. B. Collins, More Qualitative Cosmology. Comm. Math. Phys. 23 (1971), 137-158.

[11] ] A. G. Doroshkevich, V. N. Lukash, and I. D. Novikov, The isotropization of homogeneous cosmological models, Zh. Eksper. Teoret. Fiz. 64 (1973), 1457-1474. = Soviet Physics JETP 37, 739-746.

[12] V. A. Belinskii and I. M. Khalatnikov, The influence of viscosity on the character of cosmological evolution, Zh. Eksper. Teoret. Fiz. 69 (1975), 401-413. = Soviet Physics JETP 42, 205-210.

[13] I. S. Shikin, Anisotropic cosmological model in Bianchi type V in the general (axially symmetric) case with moving matter, Zh. Eksper. Teoret. Fiz. 68 (1975), 1583-1596. = Soviet Physics JETP 41, 794-800.

[14] C. B. Collins, Tilting at cosmological singularities. Comm. Math. Phys. 39 (1974), 131-151.

[15] L. I. Sedov, Metody podobiya i razmemosti v mekhanike (Methods of similarity and dimension in mechanics), Nauka, Moscow 1972.

[16] L. V. Ovsyannikov, A new solution of the equations of hydrodynamics, Dold. Akad. Nauk SSSR 111 (1957), 47-49. MR 19-89.

[17] F. J. Dyson, Dynamics of a spinning gas cloud, J. Math. Mech. 18 (1968), 91-101

[18] S. I. Anisimov and Yu. I. Lysikov, On the expansion of a gas cloud in a vacuum. Prikl. Mat. Mekh. 34 (1970), 926-930.

[19] Ya B. Zel'dovich, Newtonian and Einsteinian motion of matter, Astronom. Zh. 41 (1964), 873-883.

[20] B. Riemann, Gesammelte Werke, second ed., Teubner, Leipzig 1892; reprinted Dover, New York 1953. Translation: Sochineniya, Moscow-Leningrad 1948.

[21] S. Chandrasekkhar, Ellipsoidal figures of equilibrium, Yale Univ. Press, New Haven, Conn., 1970.

Translation: Ellipsoidal'nye figury ravnovesiya, Mir, Moscow 1973.

[22] E. M. Lifshits and I. M. Khalatnikov, Problems of relativistic cosmology, Uspekhi Fiz. Nauk 80 (1963), 391-438. = Soviet Physics Uspekhi 6 (1964), 495-522.

[23] A. H. Taub, Empty space-times admitting a three-parameter group of motions. Ann. of Math. (2) 53 (1951), 472-490. MR 12-865.

[24] V. N. Lukash, D. I. Novikov, and A. A. Starobinskii, Creation of particles in the vortex cosmological model, Zh. Eksper. Teoret. Fiz. 69 (1975), 1484-1501.

= Soviet Physics JETP 42, 757-765.

[25] O. I. Bogoyavlenskii, The qualitative theory of homogeneous cosmological models. II, Trudy Sem. Petrovskii 2 (1976).

[26] O. I. Bogoyavlenskii, The Hamiltonian formalism of a homogeneous cosmological model of type IX with an electromagnetic field, Teoret. Mat. Fiz. (1976).

[27] A. A. Peresetskii, Anisotropic homogeneous cosmological model of Bianchi type VII with motion of matter, Uspekhi Mat. Nauk 31:5 (1976), 251-252.

[28] M. Fujumoto, Gravitational collapse of rotating gaseous ellipsoids, Astrophys. J. 152 (1968), 523-537.

[29] T. Hara, T. Matsuda, and K. Nakasawe, Dynamical contraction of rotating gaseous spheroids. Progr. Theoret. Phys. 49 (1973), 460-479.

[30] D. Lynden-Bell, On the gravitational collapse of a cold rotating gas cloud, Proc. Cambridge Philos. Soc. 58 (1962), 709-711.

[31] H. Lamb, Hydrodynamics, sixth ed., Univ. Press, Cambridge 1932. Translation: Gidrodinamika, Gostekhizdat, Moscow-Leningrad 1947. 
[32] A. M. Obukhov, On integral invariants in systems ofhydrodynamical type Dokl. Akad. Nauk SSSR 184 (1969), 309-312. = Soviet Physics Dokl. 14, 32-35. 